\title{
Psychological Effect of Two-jaw Surgery
}

\author{
Shigeo Kyutoku and Yu-Ray Chen* \\ Department of Plastic Surgery, Kansal Medical University, 10-15 Moriguchi, Osaka, 570 Japan \\ Craniofacial Center, Chang-Gung Memorial Hospital*, 199 Tung-Hwa North Road, Taipei, Taiwan
}

(Received for publication October 6, 1995)

Key words: two-jaw surgery, psychological effect, psychological trauma

\section{Summary}

To know the psychological effect of two-jaw surgery, we evaluated patients' reaction to the result of the surgery which were performed to them retrospectively with questionnaire by mail. Twenty-seven out of $44(61.4 \%)$ patients, we operated between 1990 and 1991, responded to a questionnaire and most of them rated the result as satisfactory, but some shows mild disappointment especially in elder patient or big changed case. Two jaw surgery changing face significantly for adult patient is an important consideration.

\section{Introduction}

For severe jaw deformities such as cleft lip/palete midface hypoplasia, developmental jaw deformity and others, two-jaw osteotomy is performed to get normal face. The important goals of the surgery are to obtain normal occulusion, to get good intermaxillary harmony and to maintain facial midline. Though facial esthetic improvement can be easily achieved, psychological happiness may not follow. Since face is an I. D. for each individual, big change of the face is often difficult for the patient to accept and sometimes the cause of psychological trauma to body image. To investigate post-surgical psychological responces, the patients who underwent two-jaw surgery in recent one year were evaluated retrospectively with questionnaire.

\section{Material and Method}

Between January, 1990 and February, 1991, 44 patients with severe jaw deformities underwent two-jaw surgery at Craniofacial Center, Chang-Gung
Memorial Hospital (Table 1). There were 12 men and 32 women, with a mean age of 21.9 years (range 12 to 59). Presenting jaw deformities included bimaxillar protrusion (18). CL/P midface hypoplasia (17), mandibular prognathism (6) and others (3) (Table 2). Procedures have performed for these cases were Le Fort I \& sagittal splitting (12), Le Fort I \& sagittal splitting \& genioplasty (10), Wassmund \& Kole (7), Le Fort I \& Wassmund \& Kole (3) and others (12) (Table 3). The reaction of these patients as to the two-jaw surgery were evaluated retrospectively according to the questionnaire (Table 4), which were sent to all these patients by mail.

\section{Result}

Twenty-seven $(61.4 \%)$ patients completed the questionnaire and our analysis is based on these responses (Table 5). Of the 27 patients who answered, preoperative expectation is classified as expected in $6(22.2 \%)$, indifferent in $4(14.8 \%)$ and apprehensive in $17(63.0 \%)$ to it. As for the result of the surgery, $6(22.2 \%)$ rated as satisfied very 
Table 1.

44 cases of Two-Jaw Surgery (Jan.' 90 - Feb.' 91)

Table 4. Questionnaire

Name

Age.

Sex

ID

Dx

$T \mathrm{x}$

Sex $\quad M: F=12: 32$

Age $12-27 \quad(20.0)$

F $14-59 \quad(23.8)$

Table 2.

Cases for Two-Jaw Surgery M F Total

\begin{tabular}{llll}
\hline & 1 & 17 & 18 \\
bimaxillar prtrusion & 7 & 10 & 17 \\
CL/P midface hypoplasia & 4 & 2 & 6 \\
mandibular prognathism & 0 & 1 & 1 \\
henifacial microsomia & 0 & 1 & 1 \\
midface hypoplasia & 0 & 1 & 1 \\
hemimandibular hyperplasia & 0 & & \\
\hline
\end{tabular}

Table 3.

Procedures of 44 Two-Jaw Surgery

Le Fort $1+$ sagittal splitting

12

Le Fort I + sagittal splitting + genioplasty

10

Wassumund + Kole

Le Fort $1+$ Wassumund + Kole

others

Table 5. Answers for Questionnaire

\begin{tabular}{l|c|c|c|c}
\hline & satisfied & satisfied & acceptable & not \\
\hline pre & very much & & & satisfied \\
\hline expected & 1 & 2 & 3 & 0 \\
indifferent & 1 & 3 & 0 & 0 \\
apprehensive & 4 & 10 & 3 & 0 \\
\hline
\end{tabular}

Satisfied very much

Satisfied

Acceptable

Not satisfied

because_

How do you feel about the result of the surgery

you underwent? 

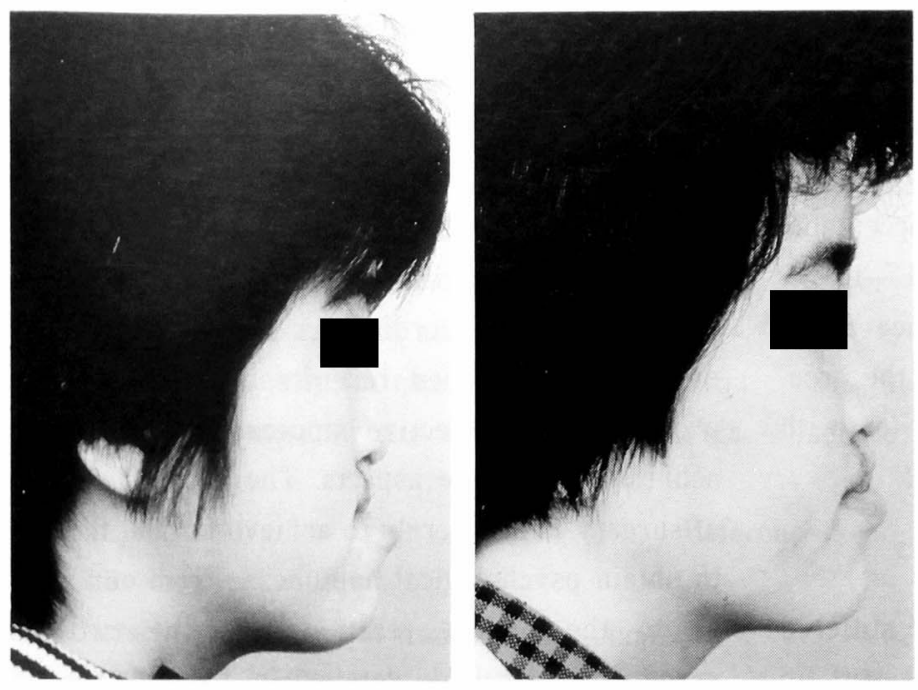

Fig. 1 Case 1. Pre- and post-operative view
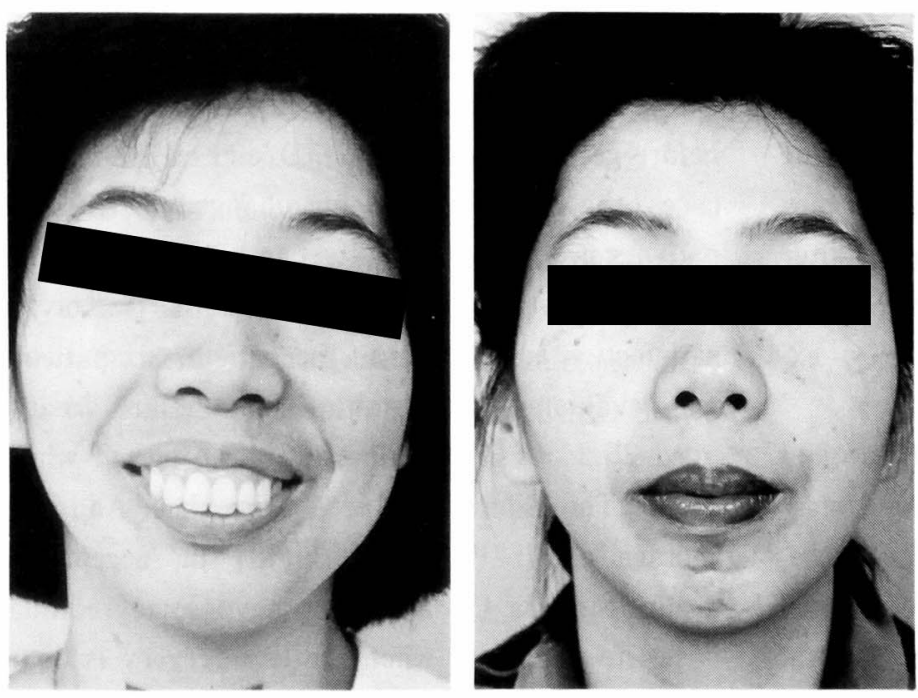

Fig. 2 Case 2. Pre- and post-operative view
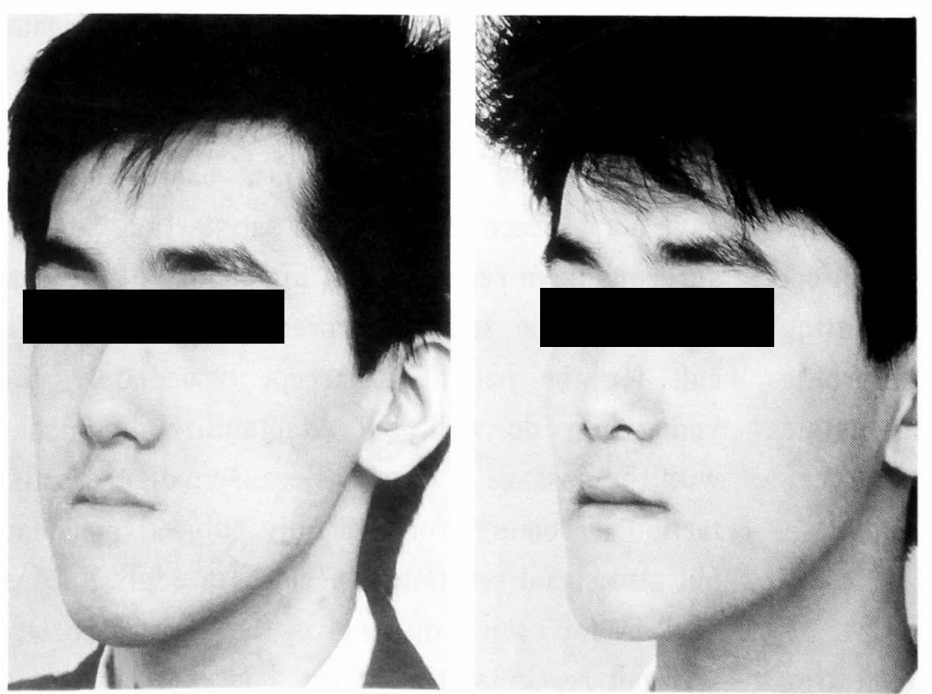

Fig. 3 Case 3. Pre- and post-operative view 
much, $15(55.6 \%)$ satisfied, $6(22.2 \%)$ acceptable and $0(0 \%)$ not satisfied. Three out of $6(50.0 \%)$ consider the result satisfied in 'expected' group and 14 out of $17(82.4 \%)$ in 'apprehensive' group. The patients who consider the result acceptable consist of 1 male and 5 female aged 17 to 31 (mean 23.2) suffered from $\mathrm{CL} / \mathrm{P}$ midface in 4 , bimaxillar prtrusion in 2 and underwent the procedure of Le Fort I \& sagittal splitting (or with genioplasty) in 4 , Wassumund \& Kole in 2.

\section{Illustrative Cases}

Case 1. A 17-year-old schoolgirl had suffered a CL/P midface hypoplasia with redidual cleft lip nasal deformity. The two-jaw surgery includes a 9-mm maxillary advancement by Le Fort I, 6-mm mandibular set back by sagittal splitting and rib bone graft onto infraorbital region. The procedure provided a good skeletal substratum for following nose repair. She was indifferent to the surgery preoperatively and thought it acceptable after surgery. Her biggest concern was nose, even if her three-dimensional skeletal improvement was excellent (Fig. 1).

Case 2. A 31-year-old housewife with a chief complaint of an anterior open bite and a gammy smile caused by bimaxillar prtrusion. The procedure includes Wassmund \& Kole to correct anterior bimaxillar prtrusion with open bite, sagittal splitting for class II malocclusion. The patient was apprehensive before surgery and rated the result satisfactory. Her postoperative profile remains her preoperative character (Fig. 2).

Case 3. A 19-year-old boy prep student presenting with severe mandibular prognathism with class III malocclusion. The surgical correction involved a $6-\mathrm{mm}$ maxillar advancement by Le Fort I, 8-mm mandibular set back by sagittal splitting and rib bone graft to infraorbital area. The profile improved excellently preserving his prognathic character intentionally. He expected the surgery and was satisfied the result very much (Fig. 3).

\section{Discussion}

For severe jaw deformities, such as cleft lip/ palete midface hypoplasia, developmental jaw deformities and bimaxillary protrusion, two-jaw osteotomy has been performed to correct abnormal physical appearance. In this kind of operations, the important goals are to obtain normal occlusion, to get good inter-maxillary harmony and to maintain facial midline. According as craniofacial techniques have developed recently, the dramatic im. provement in objective appearance can easily achieved with these aspects. The aim of this kind of surgery is not merely to achieve normal face but to obtain psychological happiness. From our study as to the patient reactions for the two-jaw surgery, the result is detailed in Table 5. Most of the patients expressed satisfactory, but the patient who said 'acceptable', which could be represent to mild disappointment, are some and deviding into two categories; one is immediate after surgery before getting used to new $\mathrm{I}$. D. especially in $\mathrm{CL} / \mathrm{P}$ cases, the other is after big changed face. These patients needed time to adjust to have new appearances and get final satisfaction. Another factor for satisfaction may be age of the patient. Six patients who evaluated the result acceptable are meanaged 23.2; it is elder than the mean age of whole group. The elders may need more time. And percentage of $\mathrm{CL} / \mathrm{P}$ case is higher in 'accentable' group. Since a $\mathrm{CL} / \mathrm{P}$ patient have some redidual nose-lip deformity when the jaw surgery is done, a profile correction might not be the biggest concern. Most of the patient would percept a change of the face two-dimensionally; profile-plasty may not be an attractive treatment. The first case, presented above, showed a mild disappointment after surgery, asking her former face back. But time gave her a peace of mind with satisfaction. Several surgeons have reported that big change of the face resulting from surgical procedures is often difficult for the patient to accept immediately; the young may do well, but adaptability diminishes with the passage of decade. Because patient's satisfaction is coming from not only subjective feeling but also social reaction, especially in adult. Face is an I. D. for each individual in the society; Society may not recognize the operated face, if its change 
is big. Fortunately, all of these 6 patients have gotten satisfied with the post-operative feature gradually, months by months. Subjective satisfaction might be assessed concomitantly after some period with social adjustment. The older, the more dependent on and involved with the society; it is almost impossible for the adult to alter the society to belong to. Consequently, in changing face, preservation of same charactaristic facial appearance is quite important besides to get 'normal face'. Illustrated latter two cases could have good physical and phychological results by preservation of some original features.

The result of this study indicate that two-jaw surgery changing face significantly for adult patient is an important consideration; procedure of two-jaw surgery could be changed with age, evaluation for the patient psycho-social condition.

\section{References}

1) Pertschuk, MJ and Whitaker, LA: Social and psychological effects of craniofacial deformity and surgical reconstruction. Clin Plast Surg, 9: 297-306, 1982.

2 ) Macgregor, FC: Social and psychological studies of plastic surgery-past, present and future. Clin Plast Surg, 9: 283-288, 1982.

3 ) Pruzinsky, T and Persing, JA: Psychological perspectives on aesthetic applications of reconstructive surgery techniques. Aesthetic Contouring of the Craniofacial Skeleton, edited by D. K. Ousterhout, pp. 43-56, Little, Brown
Co., Boston, 1991.

4) Kawamoto, Jr, HJ: Simplification of the Le Fort I osteotomy. Clin Plast Surg, 16: 777784, 1989.

5 ) Pertschuk, MJ: Psychological considerations in interface surgery. Clin Plast Surg, 18: 11$18,1991$.

6 ) Jackson, IT: Advances and applications of craniofacial surgery. Instructional Course in Annual Scientific Meeting of ASPRS/PSEF/ASMS in Seattle, September, 1991 